\title{
IMPROVED DESIGN OF FREQUENCY-RESPONSE-MASKING FILTERS USING ENHANCED SEQUENTIAL QUADRATIC PROGRAMMING
}

\author{
Wu-Sheng Lu \\ Dept. of Elec. and Comp. Engineering \\ University of Victoria \\ Victoria, BC, Canada V8W 3P6
}

\author{
Takao Hinamoto \\ Graduate School of Engineering \\ Hiroshima University \\ Higashi-Hiroshima 739-8527, Japan
}

\begin{abstract}
Sequential quadratic programming (SQP) algorithms are widely recognized to be among the most successful algorithms for nonconvex optimization. This paper attempts to develop an SQP-based method for frequency-response-masking (FRM) filters. We explain how the complementarity conditions in the SQP algorithm help reduce the amount of computation required to update the Lagrange multipliers in a significant manner. Simulation results are presented to demonstrate the algorithm's performance that compares favorably with several existing design methods.
\end{abstract}

\section{INTRODUCTION}

The frequency-response-masking (FRM) technique originated in [1] has proved to be effective for the design of digital filters with narrow transition bands. Several design methods for linear-phase and low-group-delay, FIR and IIR, basic and multistage FRM filters have been investigated in the past, see [1]-[13] and the references cited there. Among others, available design methods include joint optimization of all subfilters using semidefinite programming (SDP) [11] and second-order cone programming (SOCP) [12][13]. Although these methods work well in general, a problem with them is the large number of constraints that inevitably effects design efficiency and, in the case of high-order FRM filters, may cause numerical difficulties.

In this paper, the joint optimization of subfilters is approached in a rather different way, namely, via an enhanced sequential quadratic programming (SQP) technique. Although, to the best knowledge of the authors, it appears to be the first attempt to use SQP for the design of FRM filters, SQP algorithms are widely recognized to be among the most successful algorithms for nonconvex constrained optimization problems [14]. Since the minimax design of an FRM filter can be formulated as a nonconvex constrained minimization problem, SQP is a natural candidate tool for the design. However, our primary reason to develop an SQP-based design methodology is that the complementar- ity conditions in an SQP formulation are found to be effective in reducing the number of constraints that actually participate in the optimization. Our design method is rather general in the sense that it is applicable to both basic and multistage FRM filters with linear phase response or low group delay. Because of space limitation and for illustration clarity, however, our attention here is focused on the class of basic, linear phase FIR FRM filters. Technical details of the proposed method are given in Secs. 2 and 3. Design examples with performance comparisons are presented in Sec. 4.

\section{PROBLEM FORMULATION}

Following [1], the reader is referred to the structure in Fig. 1 where all filters are assumed to have linear-phase responses, and the lengths of the masking filters are either both even or both odd. The transfer functions of the prototype and masking filters are respectively denoted by

$$
\begin{aligned}
H_{a}(z) & =\sum_{k=0}^{N-1} h_{k} z^{-k}, H_{m a}(z)=\sum_{k=0}^{N_{a}-1} h_{k}^{(a)} z^{-k}, \\
H_{m c}(z) & =\sum_{k=0}^{N_{c}-1} h_{k}^{(c)} z^{-k}
\end{aligned}
$$

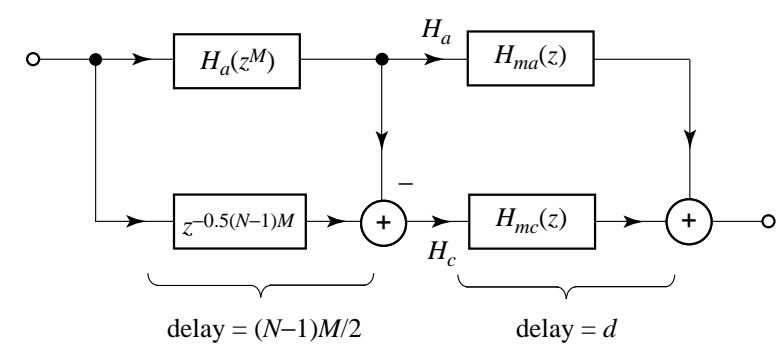

Fig. 1. A basic FRM filter structure.

In what follows, $H_{a}, H_{m a}$ and $H_{m c}$ are referred to as subfilters. Without loss of generality, the FRM filter can 
be treated as a zero-phase FIR filter and all subfilters are assumed to be of odd length. The frequency response of the FRM filter is then given by

$$
H(\omega, \boldsymbol{h})=\left[\boldsymbol{a}^{T} \boldsymbol{c}(\omega)\right]\left[\boldsymbol{a}_{a}^{T} \boldsymbol{c}_{a}(\omega)-\boldsymbol{a}_{c}^{T} \boldsymbol{c}_{c}(\omega)\right]+\boldsymbol{a}_{c}^{T} \boldsymbol{c}_{c}(\omega)
$$

where

$$
\begin{aligned}
\boldsymbol{a} & =\left[\begin{array}{llll}
h_{(N-1) / 2} & 0.5 h_{(N+1) / 2} & \cdots & 0.5 h_{N-1}
\end{array}\right]^{T} \\
\boldsymbol{c}(\omega) & =\left[\begin{array}{llll}
1 & \cos M \omega & \cdots & \cos [(N-1) M \omega / 2
\end{array}\right]^{T} \\
\boldsymbol{a}_{a} & =\left[\begin{array}{llll}
h_{\left(N_{a}-1\right) / 2}^{(a)} & 0.5 h_{\left(N_{a}+1\right) / 2}^{(a)} & \cdots & 0.5 h_{N_{a}-1}^{(a)}
\end{array}\right]^{T} \\
\boldsymbol{c}_{a}(\omega) & =\left[\begin{array}{llll}
1 & \cos \omega & \cdots & \cos \left[\left(N_{a}-1\right) \omega / 2\right.
\end{array}\right]^{T} \\
\boldsymbol{a}_{c} & =\left[\begin{array}{llll}
h_{\left(N_{c}-1\right) / 2}^{(c)} & 0.5 h_{\left(N_{c}+1\right) / 2}^{(c)} & \cdots & 0.5 h_{N_{c}-1}^{(c)}
\end{array}\right]^{T} \\
\boldsymbol{c}_{c}(\omega) & =\left[\begin{array}{llll}
1 & \cos \omega & \cdots & \cos \left[\left(N_{c}-1\right) \omega / 2\right.
\end{array}\right]^{T}
\end{aligned}
$$

and $\boldsymbol{h}=\left[\begin{array}{lll}\boldsymbol{a}^{T} & \boldsymbol{a}_{a}^{T} & \boldsymbol{a}_{c}^{T}\end{array}\right]^{T}$. The minimax design of the FRM filter amounts to finding a vector $\boldsymbol{h}$ that solves the minimax optimization problem

$$
\underset{\boldsymbol{h}}{\operatorname{minimize}}\left\{\underset{\omega \in \Omega}{\operatorname{maximize}} W(\omega)\left|H(\omega, \boldsymbol{h})-H_{d}(\omega)\right|\right\}
$$

where $H_{d}(\omega)$ is a real-valued desired frequency response, $W(\omega) \geq 0$ is a weighting function, and $\Omega=\{\omega: 0 \leq \omega \leq$ $\pi\}$.

Let $\beta$ be an upper bound of $W\left((\omega)\left|H(\omega, \boldsymbol{h})-H_{d}(\omega)\right|\right.$ on $\Omega$. As the first step of the optimization we convert the problem in (2) into a constrained minimization problem

$$
\text { minimize } \beta
$$

subject to: $W(\omega)\left|H(\omega, \boldsymbol{h})-H_{d}(\omega)\right| \leq \beta$ for $\omega \in \Omega$ (3b)

For practical exercise of optimization techniques, the constraint in (3b) is imposed on a dense grid of frequencies $\Omega_{d}=\left\{0 \leq \omega_{1} \leq \cdots \leq \omega_{K} \leq \pi\right\}$ and the problem in (3) becomes

$$
\text { minimize } \beta
$$

subject to: $W\left(\omega_{i}\right)\left|H\left(\omega_{i}, \boldsymbol{h}\right)-H_{d}\left(\omega_{i}\right)\right| \leq \beta$ for $\omega_{i} \in \Omega_{d}$

\section{DESIGN METHOD}

\subsection{An SQP-based algorithm}

The constraints in (4b) can be made more specific as

$$
\begin{aligned}
& p_{i}(\boldsymbol{h}, \beta)= \\
& \beta+W\left(\omega_{i}\right)\left[H\left(\omega_{i}, \boldsymbol{h}\right)-H_{d}\left(\omega_{i}\right)\right] \geq 0, \quad 1 \leq i \leq K \\
& p_{K+i}(\boldsymbol{h}, \beta)= \\
& \beta-W\left(\omega_{i}\right)\left[H\left(\omega_{i}, \boldsymbol{h}\right)-H_{d}\left(\omega_{i}\right)\right] \geq 0, \quad 1 \leq i \leq K
\end{aligned}
$$

Defining $\boldsymbol{x}=\left[\begin{array}{ll}\beta & \boldsymbol{h}^{T}\end{array}\right]^{T}$ and $\boldsymbol{e}=\left[\begin{array}{llll}1 & 0 & \cdots & 0\end{array}\right]^{T}$, (4) can be expressed as

$$
\begin{aligned}
& \text { minimize } e^{T} \boldsymbol{x} \\
& \text { subject to: } p_{i}(\boldsymbol{x}) \geq 0 \quad 1 \leq i \leq 2 K
\end{aligned}
$$

Since $-p_{i}(\boldsymbol{x})$ are not convex functions, (6) is a nonconvex problem.

The Lagrangian of (6) is defined by

$$
L(\boldsymbol{x}, \boldsymbol{\mu})=\boldsymbol{e}^{T} \boldsymbol{x}-\sum_{i=1}^{2 K} \mu_{i} p_{i}(\boldsymbol{x})
$$

where $\mu_{i}$ for $1 \leq i \leq 2 K$ are the Lagrange multipliers. The solution of problem (6) must satisfy the Karush-KuhnTucker (KKT) conditions [15]

$$
\begin{array}{rlrl}
\nabla L(\boldsymbol{x}, \boldsymbol{\mu}) & =\mathbf{0} & & \\
p_{i}(\boldsymbol{x}) \geq 0 & 1 \leq i \leq 2 K \\
\mu_{i} \geq 0 & 1 \leq i \leq 2 K \\
\mu_{i} p_{i}(\boldsymbol{x})=0 & 1 \leq i \leq 2 K
\end{array}
$$

It is the KKT conditions that form the basis of our design algorithm and a subsequent analysis of the algorithm. Suppose one starts with a reasonable initial point $\boldsymbol{x}_{0}$ (which may be produced using the method in [1]) and an initial $\boldsymbol{\mu}_{0}=\mathbf{0}$. In the $k$ th iteration, $\left\{\boldsymbol{x}_{k}, \boldsymbol{\mu}_{k}\right\}$ is updated to $\left\{\boldsymbol{x}_{k+1}\right.$, $\left.\boldsymbol{\mu}_{k+1}\right\}=\left\{\boldsymbol{x}_{k}, \boldsymbol{\mu}_{k}\right\}+\left\{\boldsymbol{\delta}_{x}, \boldsymbol{\delta}_{\mu}\right\}$ such that (7a), (7b), and (7c) are approximately satisfied up to the first order, and (7c) is precisely satisfied. This first-order approximation leads to

$$
\begin{aligned}
\boldsymbol{Y}_{k} \boldsymbol{\delta}_{x}+\boldsymbol{e}-\boldsymbol{A}_{k}^{T} \boldsymbol{\mu}_{k+1} & =\mathbf{0} \\
\boldsymbol{A}_{k} \boldsymbol{\delta}_{x} & \geq-\boldsymbol{c}_{k} \\
\boldsymbol{\mu}_{k+1} & \geq \mathbf{0} \\
\left(\boldsymbol{\mu}_{k+i}\right)_{i}\left(\boldsymbol{A}_{k} \boldsymbol{\delta}_{k}+\boldsymbol{c}_{k}\right)_{i} & =0 \quad 1 \leq i \leq 2 K
\end{aligned}
$$

where $\boldsymbol{Y}_{k}=\nabla^{2} L\left(\boldsymbol{x}_{k}, \boldsymbol{\mu}_{k}\right), \boldsymbol{c}_{k}=\left[p_{1}\left(\boldsymbol{x}_{k}\right) \cdots p_{2 K}\left(\boldsymbol{x}_{k}\right)\right]^{T}$, and

$$
\boldsymbol{A}_{k}=\left[\begin{array}{c}
\nabla^{T} p_{1}\left(\boldsymbol{x}_{k}\right) \\
\vdots \\
\nabla^{T} p_{2 K}\left(\boldsymbol{x}_{k}\right)
\end{array}\right]
$$

Equations (8a)-(8d) turn out to be the exact KKT conditions for the quadratic programming $(\mathrm{QP})$ problem

$$
\begin{aligned}
\operatorname{minimize} & \frac{1}{2} \boldsymbol{\delta}^{T} \boldsymbol{Y}_{k} \boldsymbol{\delta}+\boldsymbol{\delta}^{T} \boldsymbol{e} \\
\text { subject to: } & \boldsymbol{A}_{k} \boldsymbol{\delta} \geq-\boldsymbol{c}_{k}
\end{aligned}
$$

Let the solution of (10) be denoted by $\boldsymbol{\delta}_{x}$, the Lagrange multiplier $\boldsymbol{\mu}_{k+1}$ can then be determined by (8a) and (8d) as follows. First, the $2 K$ components of $\boldsymbol{A}_{k} \boldsymbol{\delta}_{x}+\boldsymbol{c}_{k}$ are examined. For the component indices with $\left(\boldsymbol{A}_{k} \boldsymbol{\delta}_{x}+\boldsymbol{c}_{k}\right)_{i}>0$, the complementarity conditions in (8d) imply that $\left(\boldsymbol{\mu}_{k+1}\right)_{i}=0$. Since $\boldsymbol{\delta}_{x}$ satisfies (10b), the rest of indices are those where $\left(\boldsymbol{A}_{k} \boldsymbol{\delta}_{x}+\boldsymbol{c}_{k}\right)_{i}=0$ and the complementarity conditions are satisfied regardless of the values of $\left(\boldsymbol{\mu}_{k+1}\right)_{i}$. These possibly 
nonzero Lagrange multipliers can be determined using (8a) as

$$
\hat{\boldsymbol{\mu}}_{k+1}=\left(\boldsymbol{A}_{a k} \boldsymbol{A}_{a k}^{T}\right)^{-1} \boldsymbol{A}_{a k}\left(\boldsymbol{Y}_{k} \boldsymbol{\delta}_{x}+\boldsymbol{e}\right)
$$

where the rows of $\boldsymbol{A}_{a k}$ are those rows of $\boldsymbol{A}_{k}$ satisfying $\left(\boldsymbol{A}_{k} \boldsymbol{\delta}_{x}+\boldsymbol{c}_{k}\right)_{i}=0$ and $\hat{\boldsymbol{\mu}}_{k+1}$ denotes the associated Lagrange multiplier. Having computed $\hat{\boldsymbol{\mu}}_{k+1}$, vector $\boldsymbol{\mu}_{k+1}$ can be obtained by inserting zeros wherever necessary in $\hat{\boldsymbol{\mu}}_{k+1}$. It should be stressed that typically the number of nonzero Lagrange multiplies, say $\hat{K}$, is much smaller than the number of constraints imposed in (4b), $K$ (usually $\hat{K}<0.1 K$ ). Consequently, computing $\hat{\boldsymbol{\mu}}_{k+1}$ using (11) which involves inversion of an $\hat{K} \times \hat{K}$ matrix does not impose a computational burden. Moreover, since in the $2 K$ linear constraints in (10b) only a small fraction of them are active, solving the QP problem in (10) can be carried out efficiently when an active-set type algorithm [15] is utilized.

Having obtained $\boldsymbol{\delta}_{x}$ and $\boldsymbol{\mu}_{k+1}$, point $\boldsymbol{x}_{k}$ is then updated to $\boldsymbol{x}_{k+1}=\boldsymbol{x}_{k}+\boldsymbol{\delta}_{x}$, and $\boldsymbol{Y}_{k}, \boldsymbol{c}_{k}$, and $\boldsymbol{A}_{k}$ are updated to $\boldsymbol{Y}_{k+1}, \boldsymbol{c}_{k+1}$, and $\boldsymbol{A}_{k+1}$, respectively. The iteration continues until a convergence criterion in terms of the progress made, i.e., $\left\|\boldsymbol{\delta}_{x}\right\|_{2}$, or the total number of iterations is met. The coefficients of the optimized subfilters can be found in the solution vector $\boldsymbol{x}^{*}$ as $\boldsymbol{h}^{*}=\boldsymbol{x}^{*}(2:$ end $)$.

\subsection{Convex relaxation of problem (10)}

The Hessian matrix of the Lagrangian, $\boldsymbol{Y}_{k}$, is in general not positive definite, hence problem (10) is not a convex QP problem. A convex relaxation of problem (10) can be made by replacing the Hessian matrix $\boldsymbol{Y}_{k}$ in (10a) with a positive definite matrix, still denoted by $\boldsymbol{Y}_{k}$, with $\boldsymbol{Y}_{0}=\boldsymbol{I}$ using the Broyden-Fletcher-Goldfarb-Shanno (BFGS) recusion [15] that updates $\boldsymbol{Y}_{k}$ to

$$
\boldsymbol{Y}_{k+1}=\boldsymbol{Y}_{k}+\frac{\boldsymbol{\eta}_{k} \boldsymbol{\eta}_{k}^{T}}{\boldsymbol{\delta}_{x}^{T} \boldsymbol{\eta}_{k}}-\frac{\boldsymbol{v}_{k} \boldsymbol{v}_{k}^{T}}{\boldsymbol{\delta}_{x}^{T} \boldsymbol{v}_{k}}
$$

where

$$
\begin{aligned}
\boldsymbol{v}_{k} & =\boldsymbol{Y}_{k} \boldsymbol{\delta}_{x} \\
\boldsymbol{\eta}_{k} & =\theta \boldsymbol{\gamma}_{k}+(1-\theta) \boldsymbol{v}_{k} \\
\boldsymbol{\gamma}_{k} & =-\left(\boldsymbol{A}_{k+1}-\boldsymbol{A}_{k}\right)^{T} \boldsymbol{\mu}_{k+1} \\
\theta & = \begin{cases}1 & \text { if } \boldsymbol{\delta}_{x}^{T}\left(\boldsymbol{\gamma}_{k}-0.2 \boldsymbol{v}_{k}\right) \geq 0 \\
\frac{0.8 \boldsymbol{\delta}_{x}^{T} \boldsymbol{v}_{k}}{\boldsymbol{\delta}_{x}^{T}\left(\boldsymbol{v}_{k}-\boldsymbol{\gamma}_{k}\right)} & \text { otherwise }\end{cases}
\end{aligned}
$$

In this way, (10) becomes a convex QP problem which possesses a unique global minimizer that can be obtained using an efficient algorithm such as an active-set algorithm. A desirable feature of the BFGS update is that if $\boldsymbol{Y}_{k}$ is positive definite, then $\boldsymbol{Y}_{k+1}$ is also positive definite. With $\boldsymbol{Y}_{0}=\boldsymbol{I}$, therefore, the QP subproblems involved in the entire design process are all guaranteed to be convex QP problems.

\subsection{Implementation}

Initial subfilters can be obtained using the method proposed in [1]. For given $H_{d}(\omega)$, weighting function $W(\omega)$, a grid of frequencies $\Omega_{d}$, and an initial $\boldsymbol{h}_{0}$, the value of $\beta_{0}$ can be calculated as

$$
\beta_{0}=\max _{\Omega_{d}} W\left(\omega_{i}\right)\left|H\left(\omega_{i}, \boldsymbol{h}_{0}\right)-H_{d}\left(\omega_{i}\right)\right|
$$

The SQP-based algorithm starts with initial point $\boldsymbol{x}_{0}=$ $\left[\begin{array}{ll}\beta_{0} & \boldsymbol{h}_{0}^{T}\end{array}\right]^{T}$ and $\boldsymbol{Y}_{0}=\boldsymbol{I}$. For the design of basic FIR FRM filters, the matrix $\boldsymbol{A}_{k}$ in (9) is a $2 K \times\left(N+N_{a}+N_{c}+5\right) / 2$ matrix whose $i$ th and $(K+i)$ th rows for $1 \leq i \leq K$ are given by $\left[1 W\left(\boldsymbol{a}_{a}^{T} \boldsymbol{c}_{a}-\boldsymbol{a}_{c}^{T} \boldsymbol{c}_{c}\right) \boldsymbol{c} W\left(\boldsymbol{a}^{T} \boldsymbol{c}\right) \bar{c}_{a} W(1-\right.$ $\left.\left.\boldsymbol{a}^{T} \boldsymbol{c}\right) \boldsymbol{c}_{c}\right]$ and $-\left[-1 W\left(\boldsymbol{a}_{a}^{T} \boldsymbol{c}_{a}-\boldsymbol{a}_{c}^{T} \boldsymbol{c}_{c}\right) \boldsymbol{c} W\left(\boldsymbol{a}^{T} \boldsymbol{c}\right) \boldsymbol{c}_{a} W(1-\right.$ $\left.\left.\boldsymbol{a}^{T} \boldsymbol{c}\right) \boldsymbol{c}_{c}\right]$ respectively, where the frequency-dependence for $W, \boldsymbol{c}, \boldsymbol{c}_{a}$, and $\boldsymbol{c}_{c}$ have been omitted. Reliable convex QP solvers are available, for example, in MATLAB Optimization Toolbox: quadprog uses an interior-point method while qp adopts an active-set method.

\section{DESIGN EXAMPLES}

The method described in Sections 2 and 3 was applied to design two one-stage linear-phase FRM filters that were addressed in the literature [1][6][11].

Example 1: The design parameters were $N=45, N_{a}=41$, $N_{c}=33, M=9, \omega_{p}=0.6 \pi, \omega_{a}=0.61 \pi, W(\omega) \equiv 1$ for $\omega \in\left[0, \omega_{p}\right] \bigcup\left[\omega_{a}, \pi\right]$ and $K=950$. It took the algorithm 110 iterations to converge to a solution FRM filter whose amplitude response and passband ripple are shown in Fig. $2 \mathrm{a}$ and $\mathrm{b}$, respectively. It is interesting to note that among the $2 K=1900$ inequality constraints (see (6b)), the average number of active constraints in the entire design process was only 27 . In other words, the average size of the matrix $\boldsymbol{A}_{a k} \boldsymbol{A}_{a k}^{T}$ in (11) was $27 \times 27$. The maximum passband ripple and minimum stopband attenuation were 0.0667 $\mathrm{dB}$ and $42.38 \mathrm{~dB}$, respectively, which compare favorably with the design of the same FRM filter in [1] (with passband ripple $=0.0896 \mathrm{~dB}$ and stopband attenuation $=40.96$ $\mathrm{dB}$ ) and in [11] (with passband ripple $=0.0674 \mathrm{~dB}$ and stopband attenuation $=42.25 \mathrm{~dB}$ ).

Example 2: The design parameters were $N=123, N_{a}=$ $56, N_{c}=78, M=21, \omega_{p}=0.4 \pi, \omega_{a}=0.61 \pi$, and $K=1100$. The weighting function $W(\omega)$ was piecewise constant with $W(\omega) \equiv 1$ in the passband and $W(\omega) \equiv 12$ in the stopband. It took the proposed algorithm 150 iterations to converge to a solution FRM filter. The average number of active constraints was 67 . The amplitude response and passband ripple of the FRM filter are depicted in Fig. 3(a) and (b), respectively. The maximum passband ripple and minimum stopband attenuation are $0.0898 \mathrm{~dB}$ and $61.66 \mathrm{~dB}$. For comparison, the maximum ripple and minimum stopband 

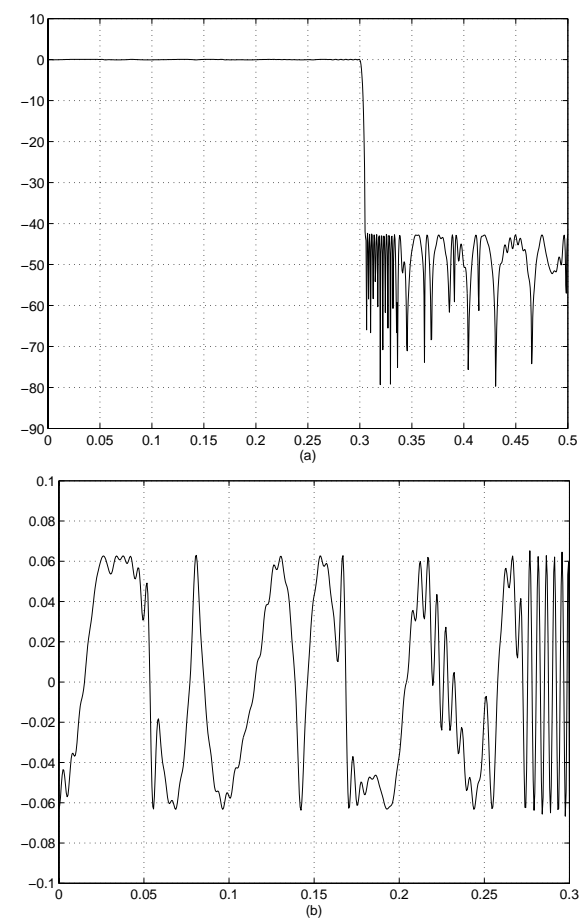

Fig. 2. (a) Amplitude response and (b) passband ripple of the FRM filter in Example 1, all in dB.

attenuation are $0.0864 \mathrm{~dB}$ and $60 \mathrm{~dB}$ in [6] and are 0.0855 $\mathrm{dB}$ and $60.93 \mathrm{~dB}$ in [11].

\section{REFERENCES}

[1] Y. C. Lim, "Frequency-response masking approach for the synthesis of sharp linear phase digital filters," IEEE Trans. Circuits Syst., vol. 33, pp. 357-364, April 1986.

[2] T. Saramaki and A. T. Fam, "Subfilter approach for designing efficient FIR filters," Proc. 1988 ISCAS, pp. 2903-2915.

[3] Y. C. Lim and Y. Lian, "The optimum design of one- and two-dimensional FIR filters using the frequency response masking technique," IEEE Trans. Circuits Syst. II, vol. 40, pp. 88-95, Feb. 1993.

[4] Y. C. Lim and Y. Lian, "Frequency-response masking approach for digital filter design: complexity reduction via masking filter factorization," IEEE Trans. Circuits Syst. II, vol. 41, pp. 518-525, Aug. 1994.

[5] T. Saramaki, Y. C. Lim, and R. Yang, "The synthesis of halfband filter using frequency-response marking technique,' IEEE Trans. Circuits Syst. II, vol. 42, pp. 58-60, Jan. 1995.

[6] T. Saramaki and H. Johansson, "Optimization of FIR filters using frequency-response masking approach," Proc. 2001 ISCAS, vol. II, pp. 177-180.

[7] L. Svensson and H. Johansson, "Frequency-response masking FIR filters with short delay," Proc. 2002 ISCAS, May 2002.
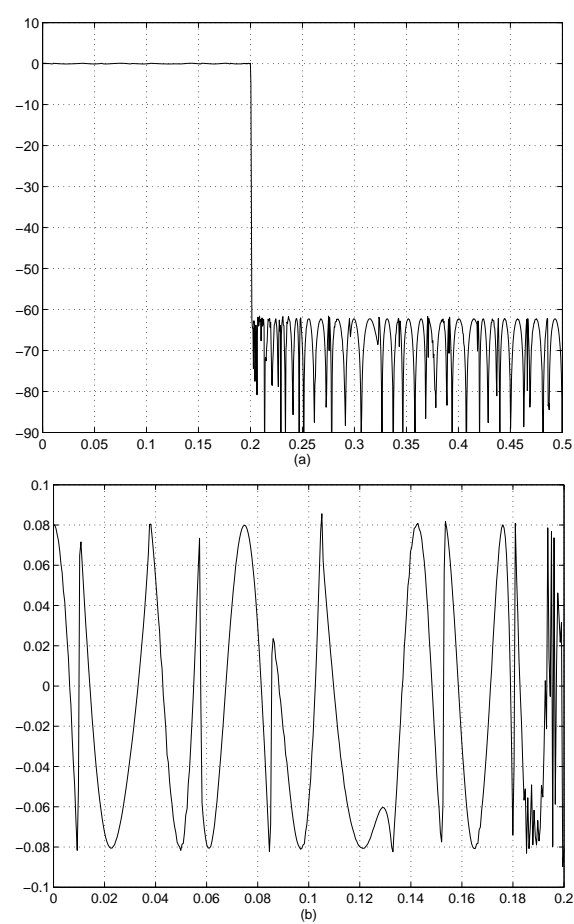

Fig. 3. (a) Amplitude response and (b) passband ripple of the FRM filter in Example 2, all in dB.

[8] T. Saramaki and Y. C. Lim, "Use of the Remez algorithm for designing FRM based FIR filters," Circuits, Systems, Signal Processing, vol. 22, no. 2, pp. 77-97, 2003.

[9] L. D. R. de Barcellos, S. L. Netto, and P. S. R. Diniz, "Optimization of FRM filters using the WLS-Chebyahev approach," Circuits, Systems, Signal Processing, vol. 22, no. 2, pp. 99-113, 2003.

[10] Y. Lian, "Complexity reduction for FRM-based FIR filters using prefilter-equalizer technique," Circuits, Systems, Signal Processing, vol. 22, no. 2, pp. 137-155, 2003.

[11] W.-S. Lu and T. Hinamoto, "Optimal design of frequencyresponse-masking filters using semidefinite programming," IEEE Trans. Circuits Syst., I, vol. 50, pp. 557-568, April 2003.

[12] W.-S. Lu and T. Hinamoto, "Optimal design of FIR frequency-response-masking filters using second-order cone programming," Proc. ISCAS'2003, vol. 3, pp. 878-881, Bangkok, May 2003.

[13] W.-S. Lu and T. Hinamoto, "Optimal design of IIR frequency-response-masking filters using second-order cone programming," to appear in IEEE Trans. Circuits Syst., I.

[14] P. T. Boggs and J. W. Tolle, "Sequential quadratic programming," Acta Numerica, vol. 4, pp. 1-51, 1995.

[15] R. Fletcher, Practical Methods of Optimization, 2nd ed., Wiley, 1987. 